University of Nebraska - Lincoln

DigitalCommons@University of Nebraska - Lincoln

Norman R. Simon Papers

Research Papers in Physics and Astronomy

$5-15-1988$

On Metallicity And RR Lyrae Light Curves

Norman R. Simon

University of Nebraska - Lincoln, nsimon@unl.edu

Follow this and additional works at: https://digitalcommons.unl.edu/physicssimon

Simon, Norman R., "On Metallicity And RR Lyrae Light Curves" (1988). Norman R. Simon Papers. 28.

https://digitalcommons.unl.edu/physicssimon/28

This Article is brought to you for free and open access by the Research Papers in Physics and Astronomy at DigitalCommons@University of Nebraska - Lincoln. It has been accepted for inclusion in Norman R. Simon Papers by an authorized administrator of DigitalCommons@University of Nebraska - Lincoln. 


\title{
ON METALLICITY AND RR LYRAE LIGHT CURVES
}

\author{
NORMAN R. SIMON \\ Department of Physics and Astronomy, University of Nebraska \\ Received 1987 August 24; accepted 1987 November 9
}

\begin{abstract}
Fourier decompositions are performed for the light curves of six $\mathbf{R R}_{a b}$ and $3 \mathbf{R} \mathbf{R}_{c}$ field stars. These are added to previous work to form a sample of $61 \mathbf{R R}_{a b}$ and $12 \mathbf{R R}_{c}$ pulsators. The $\mathbf{R}_{a b}$ stars are divided into two groups - a short-period (SP) group with $P<0.575$ and a long-period (LP) group with $P>0$.575. The Fourier phase parameter $\phi_{21}$ is found to fall off very regularly with decreasing metallicity among the SP stars, but this relationship disappears abruptly when one enters the LP group. A small sample of $\mathbf{R R}_{a b}$ stars from the globular cluster $\omega$ Cen shows hints of a similar $\phi_{21}$-metallicity relation. No trend of $\phi_{21}$ with metallicity is found for the $\mathbf{R R}_{c}$ field stars. We look for a kinematic difference between the SP and LP RR $\mathbf{R}_{a b}$ samples, but none is apparent. Finally, we use published one-zone models and new linear nonadiabatic pulsation calculations to tentatively identify the $\phi_{21}$-metallicity relation as an opacity effect. However, no explanation is found for the absence of such a relation among the long-period $\mathrm{RR}_{a b}$ stars.
\end{abstract}

Subject headings: stars: abundances - stars: pulsation - stars: RR Lyrae

\section{INTRODUCTION}

The RR Lyrae field stars comprise a wide range of metallicity and are thus of considerable interest in the study of stellar and galactic evolution. At the same time, the pulsation of these objects provides a rich source of information not available from the observation of stable stars. An attempt to link RR Lyrae light curves with metallicity was made by van Herk (1971) who found indications of a correlation between the metallicity parameter $\Delta S$ (Preston 1959) and the height of a secondary bump in the light curves for a sample of $20 \mathbf{R R}_{a b}$ stars.

The preliminary finding of van Herk was instrumental in initiating a monumental program of observations (Lub 1977a) in which more than 100 RR Lyrae field stars were observed with high accuracy and, in a majority of cases, with truly excellent coverage of the light curves. Based upon this extensive and intensive data, Lub $(1977 b)$ concluded that while "we have found indeed in several cases that a certain form of the light curve is preferred for specific abundance groups... in some cases one gets the impression that it is the period that is ruling this behavior... The initially promising results of van Herk (1971) therefore do not seem corroborated by our survey."

In the present work we use the quantitative technique of Fourier decomposition to revive the idea of a relation between metallicity and light curve structure among the field $R R_{a b}$ stars. We show further that the pulsation period plays a role in this relation, just as Lub surmised. Analysis of the data using published one-zone models gives rise to some preliminary suggestions regarding the light curve/metallicity correlation.

\section{SOME NEW FOURIER DECOMPOSITIONS}

Following Simon and Teays (1982), we treat the observations of Lub (1977a) by converting the Walraven colors to Johnson $V$ magnitudes (Pel 1976) and then fitting the light curves of each star with a Fourier series, viz.,

$$
\left.V=A_{0}+A_{j} \cos \left[j \omega\left(t-t_{0}\right)+\phi_{j}\right] \text { (sum over } j\right) .
$$

Table 1 displays Fourier decomposition data for nine stars (Lub 1977a) which were not studied by Simon and Teays
(1982). The three objects of shortest period are $\mathbf{R}_{c}$ stars, the rest $\mathbf{R R}_{a b}$ stars. The first three rows give, respectively, the period in days, the amplitude of the Johnson $V$ light curve, and the standard deviation of the Fourier fit, multiplied by 100 . The remaining rows refer to quantities defined by equation (1). For eight of the stars, the time variable entering equation (1) is

$$
t=\mathrm{JD}-2,441,000 \text {; }
$$

for the ninth star (ST Leo) the time is

$$
t=\mathrm{JD}-2,440,000 \text { (ST Leo) } \text {. }
$$

\section{METALLICITY AND LIGHT CURVE STRUCTURE}

As in many previous studies, we shall attempt here to quantify the light curve shapes in terms of combinations of the low-order Fourier coefficients, in particular, in the present case, the phase difference

$$
\phi_{21}=\phi_{2}-2 \phi_{1} \text {. }
$$

This quantity has proven very useful in treating both Cepheids (e.g., Simon and Moffett 1985) and RR Lyrae stars (e.g., Petersen 1984).

Combining the Fourier decompositions displayed in Table 1 with most of those given by Simon and Teays (1982), we have compiled a sample of $61 \mathrm{RR}_{a b}$ and $12 \mathrm{RR}_{c}$ light curves, all with high accuracy and excellent phase coverage. For each of these stars, we also have a value of the Preston metallicity parameter, $\Delta S$, taken from Lub $(1977 a)$.

We wish to examine the possibility of a correlation between $\phi_{21}$ and $\Delta S$, first for the $\mathrm{RR}_{a b}$ sample. As we shall see shortly, a raw plot of these two quantities yields no hints of a relation. However, the situation is far different when we break the $\mathbf{R R}_{a b}$ sample according to period. Figure $1 a$ shows $\phi_{21}$ versus $\Delta S$ for $37 \mathrm{RR}_{a b}$ stars in a short-period (SP) group defined by $P<$ 0.575 ; in Figure $1 b$ we display the same plot for a long-period (LP) group consisting of $20 \mathrm{RR}_{a b}$ stars with $P>0.575$. From Figures $1 a$ and $1 b$, it is clear that a striking relationship between $\Phi_{21}$ and $\Delta S$ exists for the SP group, while the LP stars show little or no evidence for such a correlation. The light 
TABLE 1

Fourier DeCOMPOSITION PARAMETers

\begin{tabular}{|c|c|c|c|c|c|c|c|c|c|}
\hline Parameter & DH Peg & YZ Cap & AU Vir & ST Leo & RY Col & RW Gru & SS Leo & DN Aqr & BH Peg \\
\hline Period (days) .......... & 0.255510 & 0.27345698 & 0.3432302 & 0.4779843 & 0.4788571 & 0.550273 & 0.6263432 & 0.6335279 & 0.6410009 \\
\hline Amplitude (mag) ...... & 0.506 & 0.527 & 0.535 & 1.34 & 0.722 & 1.11 & 1.18 & 0.795 & 0.693 \\
\hline$\sigma(\times 100) \ldots \ldots \ldots \ldots \ldots$ & 1.51 & 2.29 & 2.44 & 2.99 & 3.02 & 3.30 & 3.73 & 2.33 & 2.01 \\
\hline$t_{0} \quad \ldots \ldots \ldots \ldots \ldots \ldots \ldots$ & 129.0 & 856.0 & 794.0 & 648.0 & 694.0 & 208.0 & 741.0 & 132.0 & 216.0 \\
\hline$A_{0} \quad \ldots \ldots \ldots \ldots \ldots \ldots \ldots$ & 9.57 & 11.33 & 11.63 & 11.58 & 10.98 & 12.48 & 11.10 & 12.50 & 10.47 \\
\hline$A_{1} \quad \ldots \ldots \ldots \ldots \ldots \ldots \ldots$ & $2.47(-1)$ & $2.43(-1)$ & $2.42(-1)$ & $4.36(-1)$ & $3.00(-1)$ & $4.02(-1)$ & $3.80(-1)$ & $2.76(-1)$ & $2.67(-1)$ \\
\hline$\phi_{1} \quad \ldots \ldots \ldots \ldots \ldots \ldots$ & 5.00 & 2.32 & 3.91 & 2.11 & 3.95 & 4.65 & 5.42 & 2.05 & 3.81 \\
\hline$A_{2} \quad \ldots \ldots \ldots \ldots \ldots \ldots \ldots$ & $4.58(-2)$ & $4.32(-2)$ & $3.75(-2)$ & $2.18(-1)$ & $1.22(-1)$ & $1.84(-1)$ & $2.03(-1)$ & $1.24(-1)$ & $9.11(-2)$ \\
\hline$\phi_{2} \quad \ldots \ldots \ldots \ldots \ldots \ldots \ldots$ & 2.12 & 2.90 & 6.15 & 1.82 & 5.78 & $4.73(-1)$ & 2.21 & 1.99 & 5.83 \\
\hline$A_{3} \quad \ldots \ldots \ldots \ldots \ldots \ldots$ & $1.36(-2)$ & $1.60(-2)$ & $2.56(-2)$ & $1.54(-1)$ & $7.93(-2)$ & $1.32(-1)$ & $1.25(-1)$ & $8.62(-2)$ & $4.34(-2)$ \\
\hline$\phi_{3} \quad \ldots \ldots \ldots \ldots \ldots \ldots \ldots$ & 5.35 & 3.58 & 2.25 & 1.81 & 1.18 & 3.08 & 5.65 & 2.07 & 1.29 \\
\hline$A_{4} \quad \cdots \cdots \cdots \cdots \cdots \cdots$ & $1.77(-2)$ & $1.85(-2)$ & $1.98(-2)$ & $9.88(-2)$ & $4.04(-2)$ & $7.03(-2)$ & $9.55(-2)$ & $5.24(-2)$ & $1.40(-2)$ \\
\hline$\phi_{4} \quad \ldots \ldots \ldots \ldots \ldots \ldots \ldots$ & 2.56 & 4.45 & 5.03 & 1.88 & 3.32 & 5.57 & 2.81 & 2.42 & 4.68 \\
\hline$A_{5} \quad \ldots \ldots \ldots \ldots \ldots \ldots \ldots$ & $\ldots$ & $\ldots$ & $\ldots$ & $7.88(-2)$ & $2.55(-2)$ & $6.35(-2)$ & $5.40(-2)$ & $3.06(-2)$ & $2.05(-2)$ \\
\hline$\phi_{5} \quad \cdots \cdots \cdots \cdots \cdots \cdots$ & $\ldots$ & $\cdots$ & $\ldots$ & 1.98 & 5.60 & 1.82 & $8.30(-1)$ & 2.87 & 1.32 \\
\hline$A_{6} \quad \cdots \cdots \cdots \cdots \cdots \cdots$ & $\ldots$ & $\ldots$ & $\cdots$ & $4.61(-2)$ & $2.41(-2)$ & $3.41(-2)$ & $3.21(-2)$ & $1.44(-2)$ & $1.63(-2)$ \\
\hline$\phi_{6} \quad \ldots \ldots \ldots \ldots \ldots \ldots \ldots$ & $\ldots$ & $\ldots$ & $\ldots$ & 2.17 & 1.09 & 4.76 & 3.38 & 3.25 & 3.75 \\
\hline$A_{7} \quad \ldots \ldots \ldots \ldots \ldots \ldots$ & $\ldots$ & $\ldots$ & $\ldots$ & $3.05(-2)$ & $1.49(-2)$ & $2.42(-2)$ & $2.22(-2)$ & $1.58(-2)$ & $9.92(-3)$ \\
\hline$\phi_{7} \quad \ldots \ldots \ldots \ldots \ldots \ldots \ldots$ & $\ldots$ & $\ldots$ & $\ldots$ & 2.26 & 3.50 & $5.65(-1)$ & 6.14 & 4.08 & 5.69 \\
\hline$A_{8} \quad \ldots \ldots \ldots \ldots \ldots \ldots \ldots$ & $\ldots$ & $\ldots$ & $\ldots$ & $2.35(-2)$ & $1.27(-2)$ & $1.37(-2)$ & $1.71(-2)$ & $1.25(-2)$ & $7.59(-3)$ \\
\hline$\phi_{8} \quad \cdots \cdots \cdots \cdots \cdots \cdots \cdots$ & $\ldots$ & $\ldots$ & $\ldots$ & 1.87 & 5.31 & 3.42 & 4.24 & 3.97 & 1.38 \\
\hline
\end{tabular}

curves in the present sample are so accurate that the error in determining $\phi_{21}$ is, for our purposes, negligible. However, there is an observational uncertainty in $\Delta S$, estimated by Preston $(1959)$ as $\delta(\Delta S) \approx 2$. Taking this into account, one sees that the $\phi_{21}-\Delta S$ correlation among the SP group is probably even tighter than implied by Figure $1 a$.

The period range of the $\mathbf{R R}_{a b}$ stars plotted in Figures $1 a$ and $1 b$ is $0.374 \leq P \leq 0$ d 747 . The boundary between the SP and
LP groups $(P \approx 0$ d.575) is not defined precisely, since the SP stars include one object with a slightly larger period (V341 Aql; $P=0.578)$, and the LP stars include one object with a slightly shorter period (RV Oct; $P=0.571$ ). The assignment of these two stars to their respective groups was made on the basis of their values of $\phi_{21}$ and $\Delta S$. Thus, in summary, the SP group consists of 36 stars all with $P<0$ d.575, plus V341 Aql, while in the LP group there are 19 stars all with $P>0.575$, plus RV
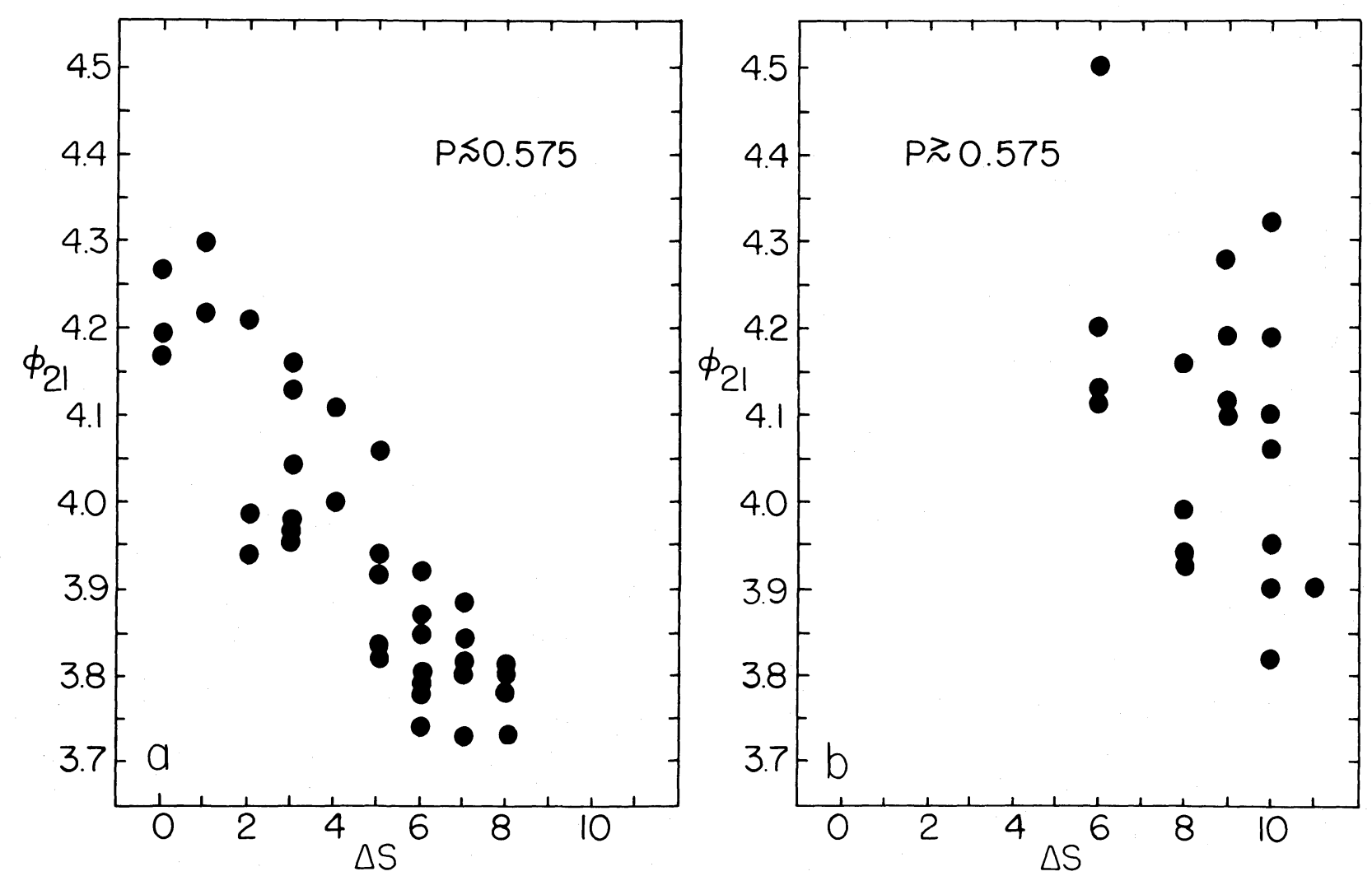

Fig. 1.- $\phi_{21}$ vs. $\Delta S$ for the $\mathrm{RR}_{a b}$ sample. (a) Short-period (SP) group; (b) long-period (LP) group. 
Oct. Finally one notices from Figures $1 a$ and $1 b$ that the SP stars comprise a range of metallicity $0 \leq \Delta S \leq 8$, and the LP stars $6 \leq \Delta S \leq 11$.

In Figure 2 we have plotted $\phi_{21}$ versus $\Delta S$ for the combined sample of both the SP stars (dots) and the LP stars (crosses). While their separation appears clearly on this diagram, it is also apparent that the SP correlation might easily have been missed if no period separation had been effected. The four open circles in Figure 2 denote stars which do not seem to fit the general picture. At the lower left are AA Aql and S Ara: they belong to the SP group, according to period, but lie below the $\phi_{21}-\Delta S$ relation. The other two discrepant stars, UU Cet and FY Hya, seem to follow the SP relation but have periods $(0.606$ and 0.637 , respectively) which place them well within the LP group. We emphasize that Figure 2 displays the entire $\mathrm{RR}_{a b}$ sample of 61 stars. We used every available object with a first-rate light curve and a published value of $\Delta S$. No prior selection was made to produce the results shown in Figures 1 and 2.

Simon and Aikawa (1986) demonstrated for the $\mathbf{R R}_{a b}$ field stars, a loose correlation between $\phi_{21}$ and the amplitude. Figure 3 shows a plot of $V$ amplitude versus $\phi_{21}$ for the SP sample. We have distinguished two different metallicity groups in this diagram: $0 \leq \Delta S \leq 4$, plotted as dots, and $5 \leq \Delta S \leq 8$, plotted as crosses. These two groups certainly seem to display different slopes, with the higher metallicity component (smaller $\Delta S$ ) lying above the lower metallicity component. For the LP stars (not plotted), the amplitude is also seen to fall off with $\phi_{21}$, as in Simon and Aikawa (1986), but there is no distinction

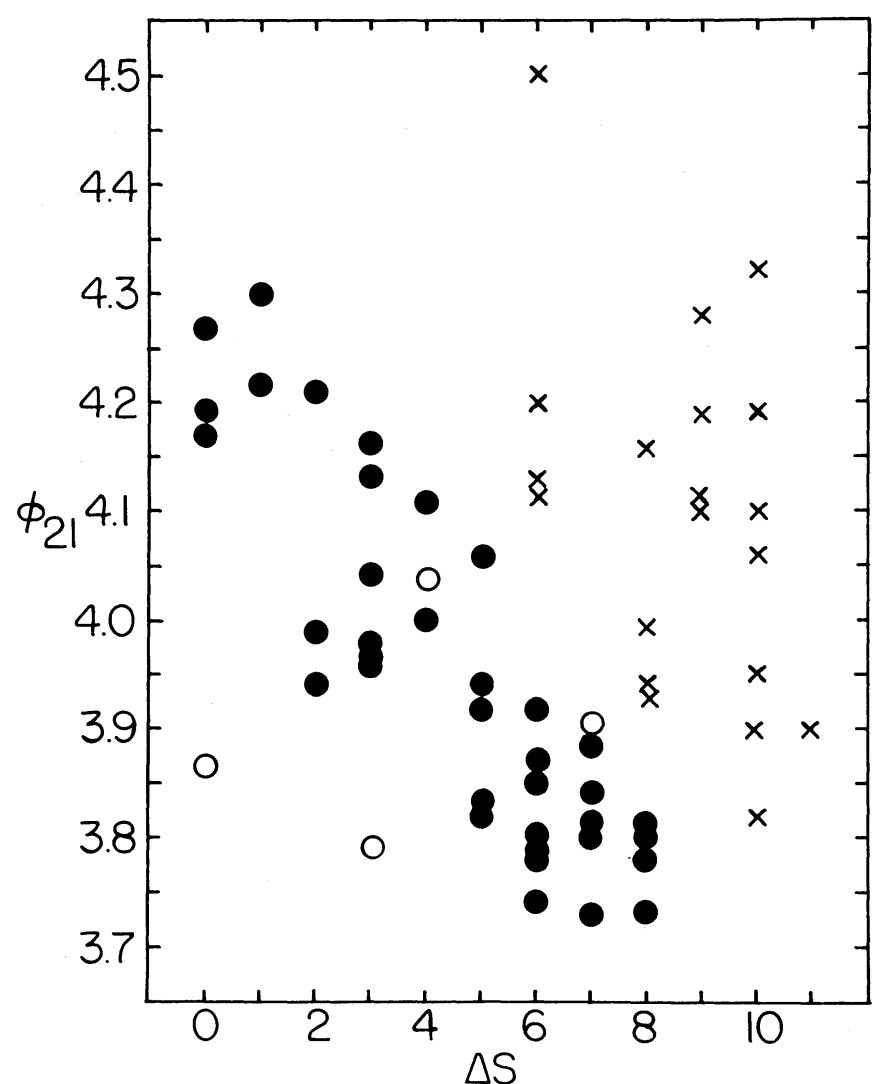

FIG. $2 .-\phi_{21}$ vs. $\Delta S$ for the entire RR $R_{a b}$ sample. (dots): SP stars; (crosses): LP stars; (open circles): discrepant stars.

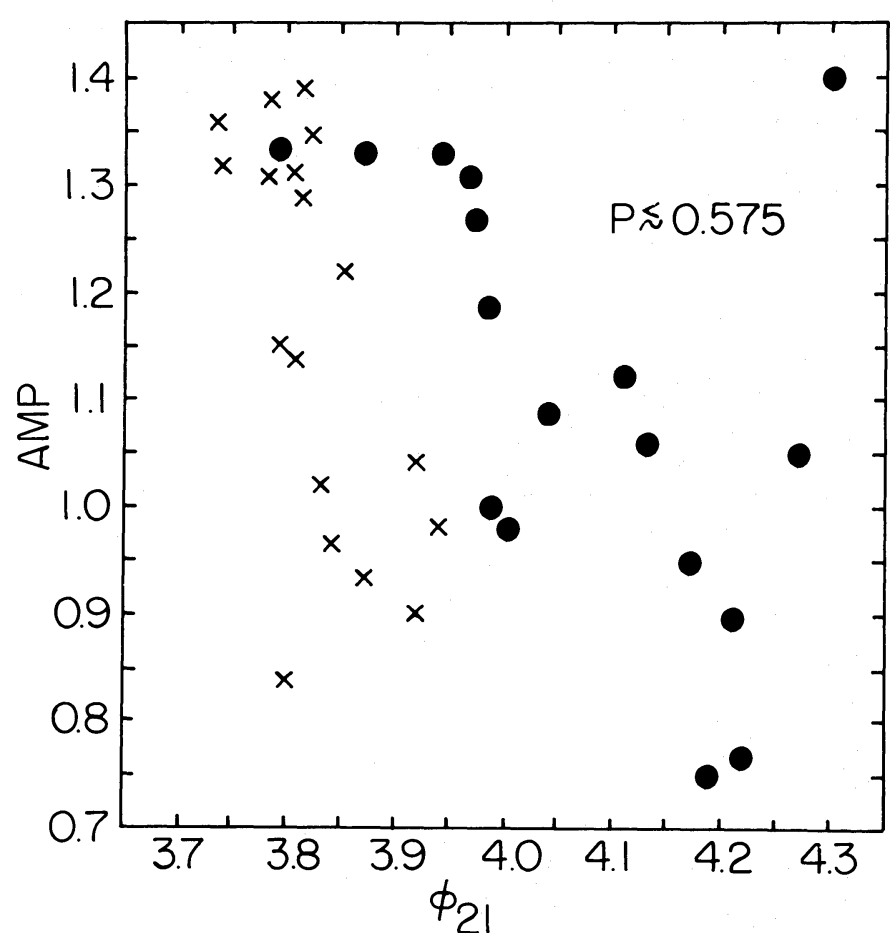

FIG. 3. $-V$ amplitude vs. $\phi_{21}$ for the SP stars. (dots): $0 \leq \Delta S \leq 4$; (crosses): $5 \leq \Delta S \leq 8$.

noticeable among different metallicities. Finally, we plot in Figure 4 the $V$ amplitude versus $\Delta S$ for both the SP (dots) and LP (crosses) components. No correlation between amplitude and metallicity is apparent in either group. However, where the metallicities overlap, the SP stars tend to have larger amplitudes than the LP stars at given $\Delta S$.

We close this section with some remarks on the correspondence between Fourier phases and light curve shapes. Simon (1985), using light curves generated from synthetic Fourier series, and Stellingwerf and Donohoe (1987), employing onezone models, reached the same conclusion-namely, that the effect of the Fourier phases could be described in terms of the width (or, alternatively, the narrowness, or "acuteness") of the light curve at half-maximum light. The sense of this correspondence is that as the phase differences narrow, so does the breadth of the light curve at half maximum. Thus, a smaller value of $\phi_{21}$ implies a more acute light curve. The synthetic light curves plotted by Simon (1985) also indicate that changes in the Fourier phases produce alterations in the shape and location of "bumps" which appear during descending light. However, it is not clear if the correlation presented in Figure $1 a$ of the present work is in any way related to the qualitative or semiquantitative correspondence between bumps and metallicity suggested by van Herk (1971).

\section{RR LYRAE STARS IN $\omega$ CENTAURI}

In view of the $\phi_{21}-\Delta S$ relation that exists among the SP sample of $\mathbf{R R}_{a b}$ field stars, it is interesting to inquire whether similar trends may be found in globular cluster pulsators. The rich cluster $\omega$ Cen immediately comes to mind due to the large spread in metallicity among its stars. Petersen (1984) performed Fourier decomposition on the photographic light curves of $75 \mathbf{R R}_{a b}$ stars from the $\omega$ Cen observations of Martin (1938) and published Fourier coefficients for 20 of these. In a 


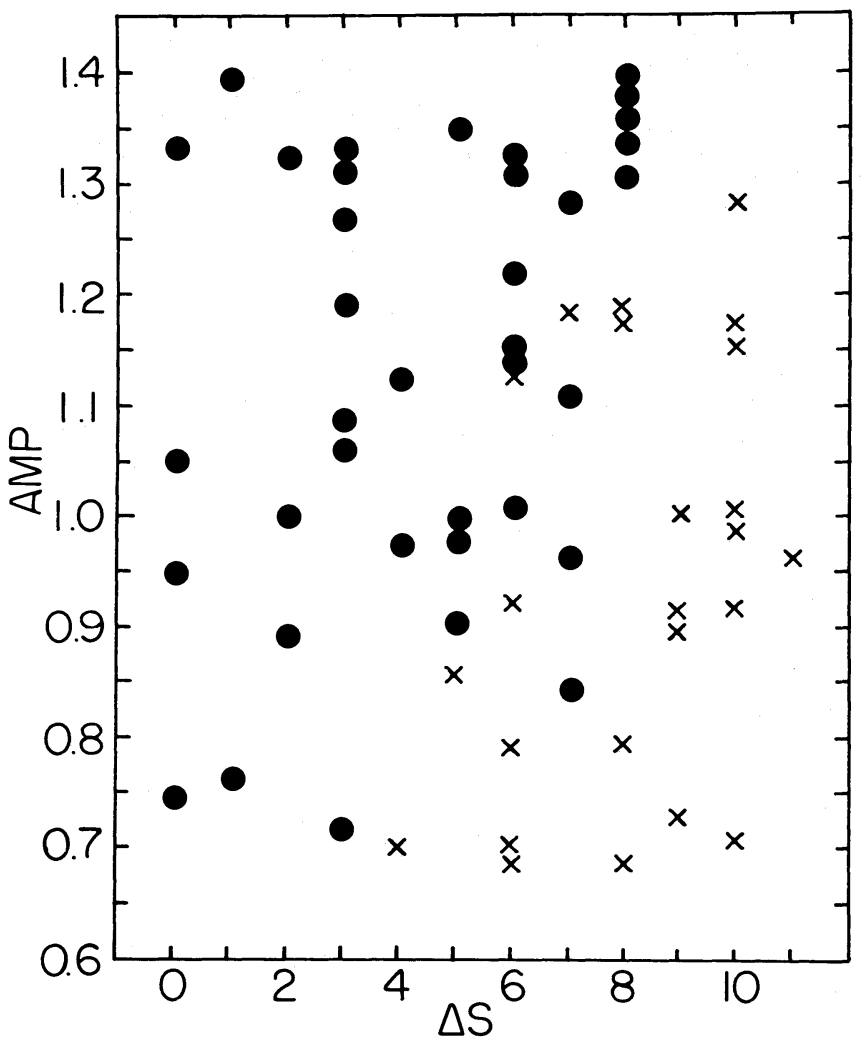

Fig. 4. $-V$ amplitude vs. $\Delta S$. (dots): SP stars; (crosses): LP stars.

search of the article by Butler, Dickens, and Epps (1978), we were able to find $[\mathrm{Fe} / \mathrm{H}]$ values for half of the 20 stars. These values were converted to $\Delta S$ values using the approximate relation (Lub 1977b),

$$
[\mathrm{Fe} / \mathrm{H}]=-0.14-0.175 \Delta S .
$$

Figure 5 shows a plot of $\phi_{21}$ versus $\Delta S$ for the $10 R_{a b}$ stars we were able to treat in $\omega \mathrm{Cen}$. One notices the possibility of a tread similar to that among the field stars. (Note that it is necessary to augment $\phi_{21}$ by an amount $\sim 0.15$ to convert the photographic data to an equvalent in the $V$ magnitude [Petersen and Hansen 1984; Simon and Moffett 1985]. Had we done this in Fig. 5, the range of $\phi_{21}$ values would have been more similar to that displayed in Figs. 1 and 2.) However, the small sample we used encompassed a very narrow period range $(0.50-0.58)$ and needs to be augmented by other stars held in common by the Petersen (1984) and Butler, Dickens, and Epps (1978) investigations. Unfortunately, this cannot be done with presently published data.

\section{DISCUSSION}

The analysis in previous sections suggests that it is useful to group the field $\mathrm{RR}_{a b}$ stars according to period. The SP stars $(P<0$ d.575) show a definite relationship between light curve shape and metallicity, while the LP stars $(P>0$ d.575) do not. In this circumstance it is tempting to suggest that the two groups may have different origins. One way of getting at this question is to examine the kinematic properties of the stars. We have done this using the observational data published by van Herk (1965). A cursory, nonstatistical analysis indicates no evidence of any difference between the SP and LP stars with regard to either location or motion in the Milky Way. For example, Figure 6 shows a plot of velocity in the direction of the galactic rotation (van Herk 1965) versus period for all of the stars in our sample with $\Delta S \geq 5$. (We chose here the more metal-poor stars, since it is already well known that the metal-rich and metal-poor stars are kinematically different, and it is not this distinction that we seek.) The SP stars are plotted as dots; the LP stars, as crosses. No hint of a kinematic difference appears.

How then is one to explain the dichotomy displayed in Figures 1 and 2 and, in particular, the strong relation between $\phi_{21}$ and $\Delta S$ shown in Figure $1 a$ ? The ultimate arrival of an RR Lyrae star in the instability strip is governed by its own interior evolution and by mass loss. If one assumes that stars with similar light curves have similar interior structures and locations in the strip, then the tight relation between $\phi_{21}$ and $\Delta S$ among the SP group would seem to imply that both the interior evolution of these stars and the amount of mass loss depend principally (and rather precisely) on the metallicity. Indeed, Butler, Dickens, and Epps (1978) have presented evidence for just such a dependence among the $\mathbf{R R}_{a b}$ stars in the globular cluster $\omega$ Cen.

However, the $\phi_{21}-\Delta S$ trend in the SP field stars encompasses a large range of metallicity, $0 \leq \Delta S \leq 8$, and thus presumably includes both old-disk and halo objects. Because these stars have different ages and, supposedly, very different histories (accounting for the metal-rich RR Lyraes is a difficult problem

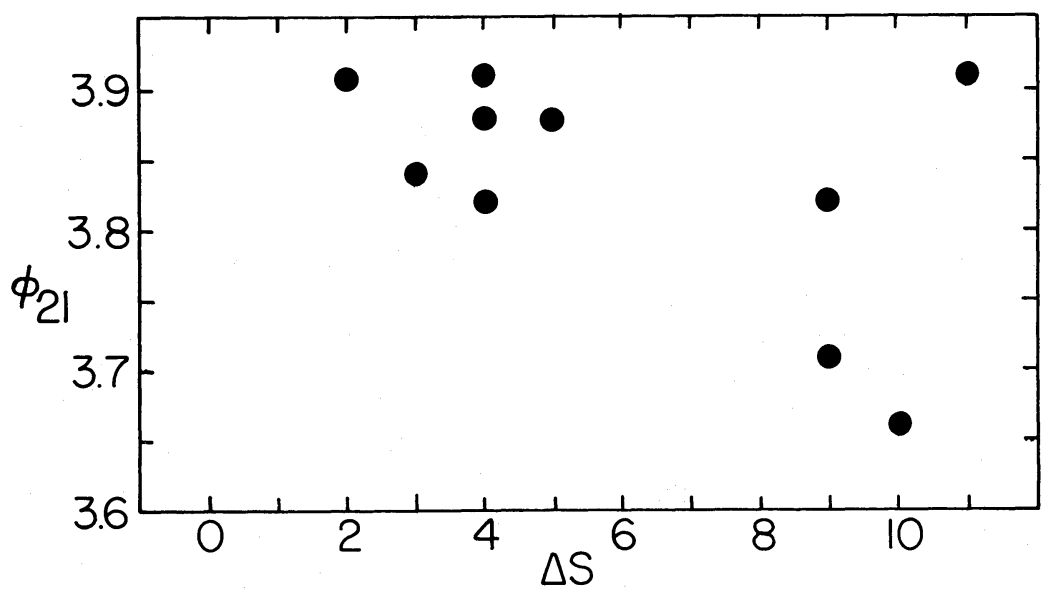

FIG. 5. $-\phi_{21}$ vs. $\Delta S$ for photographic light curves of $10 \mathrm{RR}_{a b}$ stars in $\omega$ Cen 


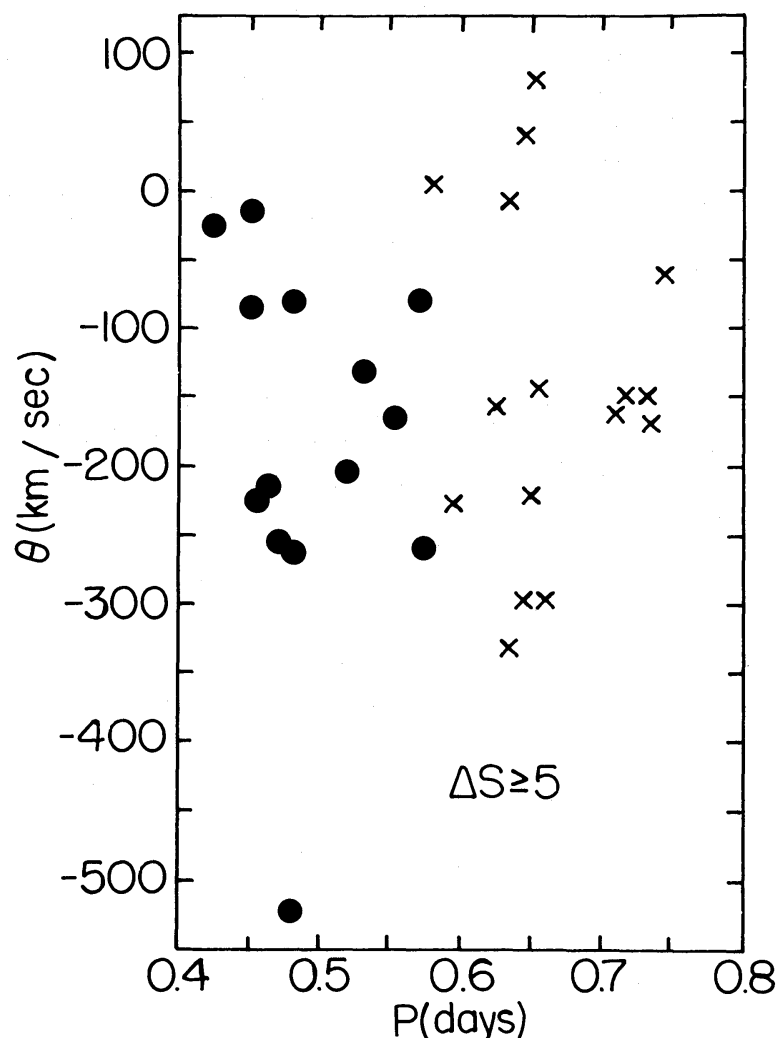

FIG. 6.-Velocity in direction of galactic rotation vs. period for $\mathbf{R R}_{a b}$ stars with $\Delta S \geq 5$. (dots): SP stars; (crosses): LP stars. in itself; see, e.g., Taam, Kraft, and Suntzeff 1976), it is hard to see how they could be so well orchestrated by a single parameter (metallicity) that they would all follow the tight relation displayed in Figure 1a. (For a similar, and perhaps related, problem involving the short-period type II Cepheids, see Simon 1986). This picture is further confused by the lack of any correlation between $\phi_{21}$ and the pulsation period among the SP stars (Simon and Teays 1982).

Suppose, however, that the value of $\phi_{21}$ for a given RR Lyrae light curve is not determined by the global parameters of the star, but rather, locally, i.e., it depends upon the physical conditions at some particular place in the envelope (see also Aikawa, Antonello, and Simon 1987). Then two stars with similar values of $\phi_{21}$ need not have a similar mass, luminosity, or period, but only a similar condition in the critical region of the envelope. Then, to complete this picture, the physical state of the critical region would be determined by the metal abundance. We will examine this idea in what follows.

In Figure 7 we plot the Fourier phase difference $\phi_{31}=$ $\phi_{3}-3 \phi_{1}$ (see eq. [1]) vs. $\Delta S$ for both the SP (dots) and LP (crosses) samples. We notice that both the separation between the two groups and, particularly, the trend among the SP stars are much less distinct than was the case for $\phi_{21}$ (Fig. 2). Again, we remark that the light curves we have treated are so well defined that the errors in determining even the higher order term $\phi_{31}$ are very small. Thus almost all of the additional scatter in Figure 7 (as cmpared with Fig. 2) is real. This result is consistent with the idea that $\phi_{31}$ is determined in a somewhat different regior of the envelope, one that is perhaps not quite so narrow nor so influenced by the metallicity as is the critical region which fixes $\phi_{21}$.

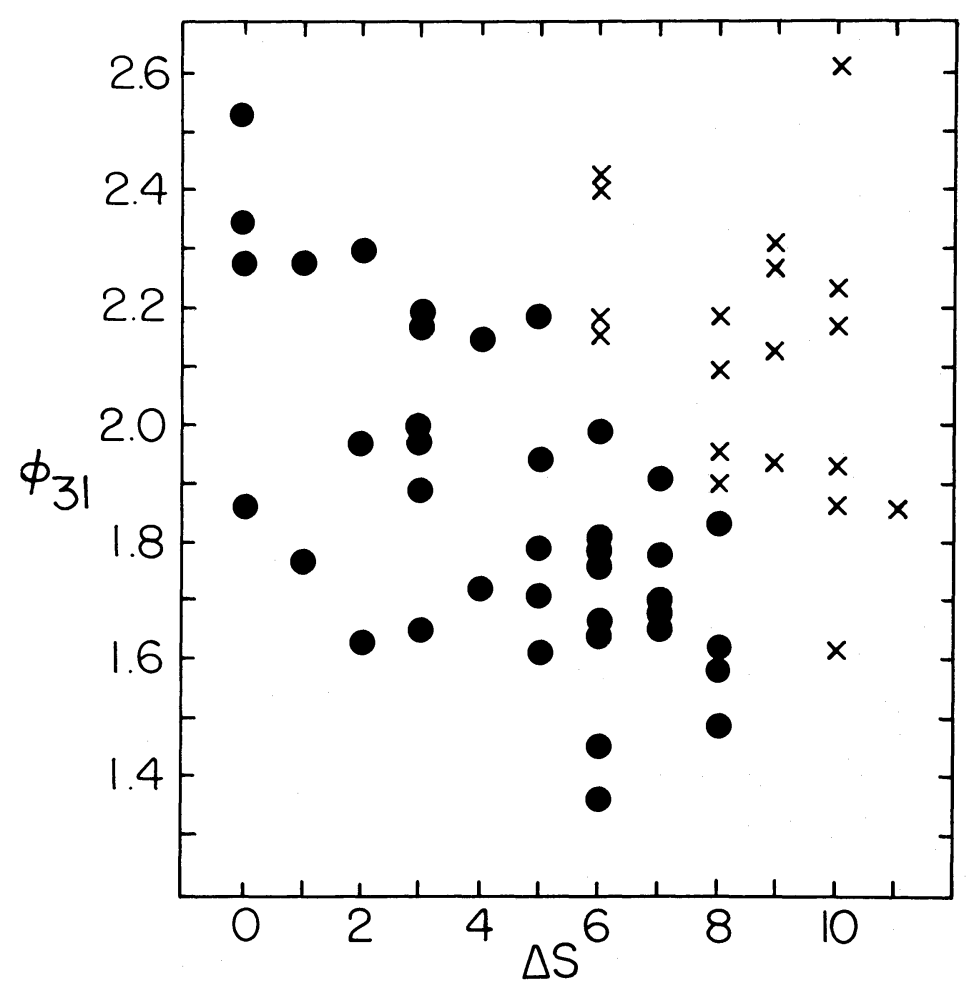

FIG. 7. $-\phi_{31}$ vs. $\Delta S$ for the $\mathrm{RR}_{a b}$ sample. (dots): SP stars; (crosses): LP stars. 


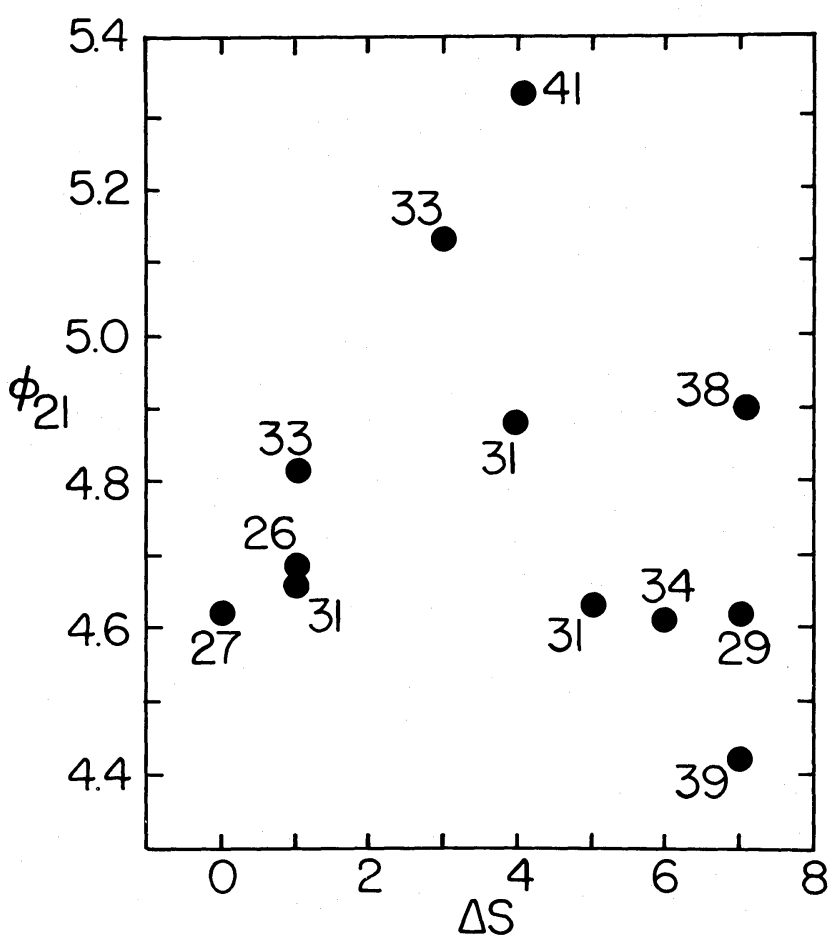

FIG. 8. $-\phi_{21}$ vs. $\Delta S$ for the $\mathrm{RR}_{c}$ stars. Period in days (times 100) is indicated for each star.

A similar surmise may be drawn from Figure 8 in which we have plotted $\phi_{21}$ versus $\Delta S$ for the $12 \mathrm{RR}_{c}$ stars in our sample. The number beside each point gives the period in days (times 100). The data treated here (though, admittedly not extensive) provide little evidence for either a selection by period among the $\mathrm{RR}_{c}$ stars nor for a trend of $\phi_{21}$ with $\Delta S$ similar to that among the $\mathbf{R R}_{a b}$ stars. Once more, this result would be expected if, as is likely, $\phi_{21}$ for the first overtone is determined in a different region than is $\phi_{21}$ for the fundamental mode.

Some hint as to how the metallicity might determine $\phi_{21}$ may be obtained from the work of Stellingwerf and Donohoe (1987, hereafter SD) who constructed a grid of nonlinear, onezone pulsation models. These authors found $\phi_{21}$ to be a function of the size of the luminosity amplitude relative to the radius amplitude in the region where the pulsation becomes adiabatic, just below the ionization zones. In the one-zone models, the quantity $u$ (defined by SD as a measure of the luminosity amplitude below the pulsating shell) is seen to depend on the temperature exponent of the opacity, i.e., $\kappa_{T} \equiv$ $(\partial \log \kappa / \partial \log T)_{\rho}$.

To examine this idea further we have constructed a series of linear, nonadiabatic (LNA) RR Lyrae pulsation models with various masses, luminosities, and temperatures. In all of these models there appears, below the ionization regions, a zone in which the opacity temperature exponent achieves a relative maximum in absolute value (i.e., $\kappa_{T}$, which is negative, achieves a minimum). The temperature in this zone lies in the range $1.75-2.00 \times 10^{5} \mathrm{~K}$; this is just the region in which the pulsation is establishing itself as strongly adiabatic (region of adiabatic onset). Furthermore, the strength of the maximum attained by $\left|\kappa_{T}\right|$ is found to depend on the metallicity in such a way that the smaller the value of $Z$ for a given model, the larger the value of $\left|\kappa_{T}\right|_{\text {max }}$. This last finding is easily explained as follows: The heavy element opacity is less sensitive to temperature than is the opacity due to hydrogen and helium. Thus when $Z$ is larger, and, consequentially, the metal opacity is relatively more important, the temperature exponent $\left|\kappa_{T}\right|$ is reduced.

It is also possible to calculate from the LNA models a quantity which measures the relative strength of the luminosity amplitude as introduced by SD. The LNA parameter corresponding to that defined for the one-zone models is

$$
u=\frac{\left|\delta L_{r} / L_{r}\right|}{|\delta r / r|}
$$

i.e., the ratio of the normalized luminosity amplitude to the normalized radius amplitude. We find that $u$ attains a maximal value at approximately the same place as $\left|\kappa_{T}\right|$ and that $u_{\max }$ is an increasing function of $\left|\kappa_{T}\right|_{\max }$.

Figure 9 portrays the LNA results for a single series of models with $M=0.55 M_{\odot}, L=39.17 L_{\odot}, T_{e}=6600 \mathrm{~K}$, $Y=0.20, P_{0}=0$ d.52. The models differ only in metallicity $Z$. We plot $\left|\kappa_{T}\right|_{\max }$ and $u_{\max }$ (note the scales decreasing upward) versus $\log Z$. It is clear that both these quantities increase with decreasing metal abundance. This is true generally for all our models. Furthermore, an examination of the LNA calculations shows that the value of $u_{\max }$ may be taken as an indicator of the strength of $u$ in the region of adiabatic onset. That is to say, the

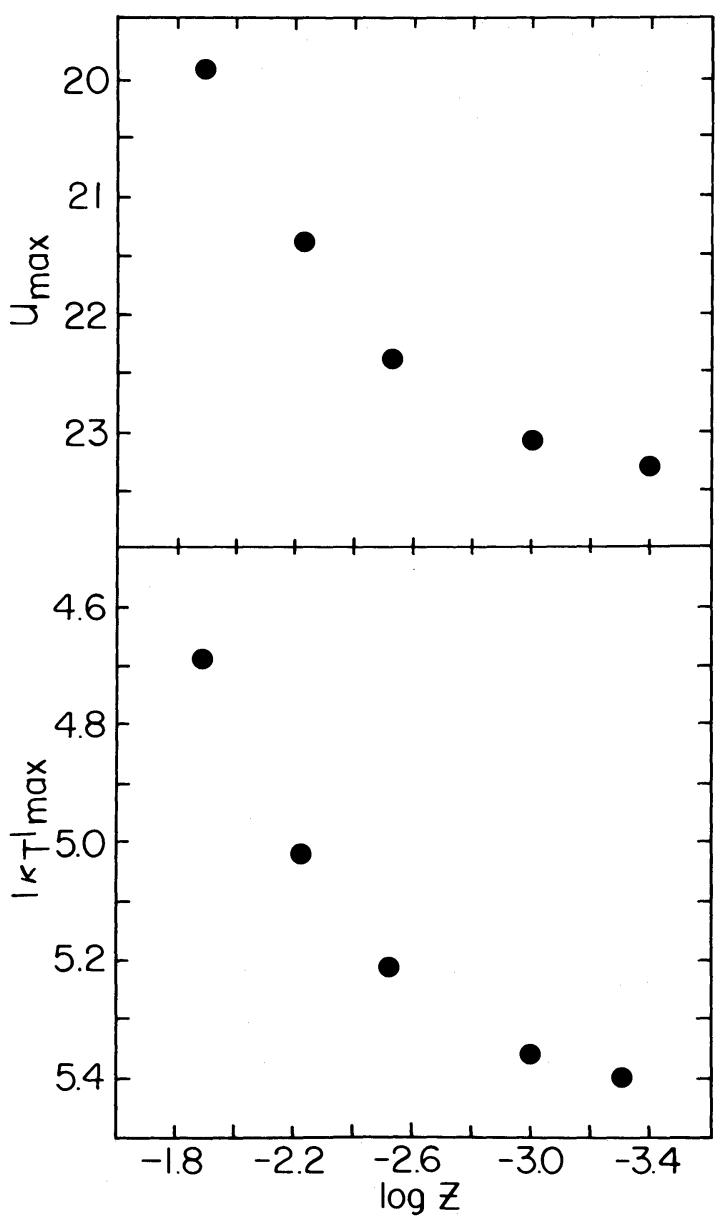

Fig. 9. $-u_{\max }$ and $\left|\kappa_{T}\right|_{\max }$ vs. $\log Z$ for LNA pulsation models with $M=0.55 M_{\odot}, L=39.17 L_{\odot}, T_{e}=6600 \mathrm{~K}, Y=0.20, P_{0}=0.52$. Note the ordinate decreasing upward. 


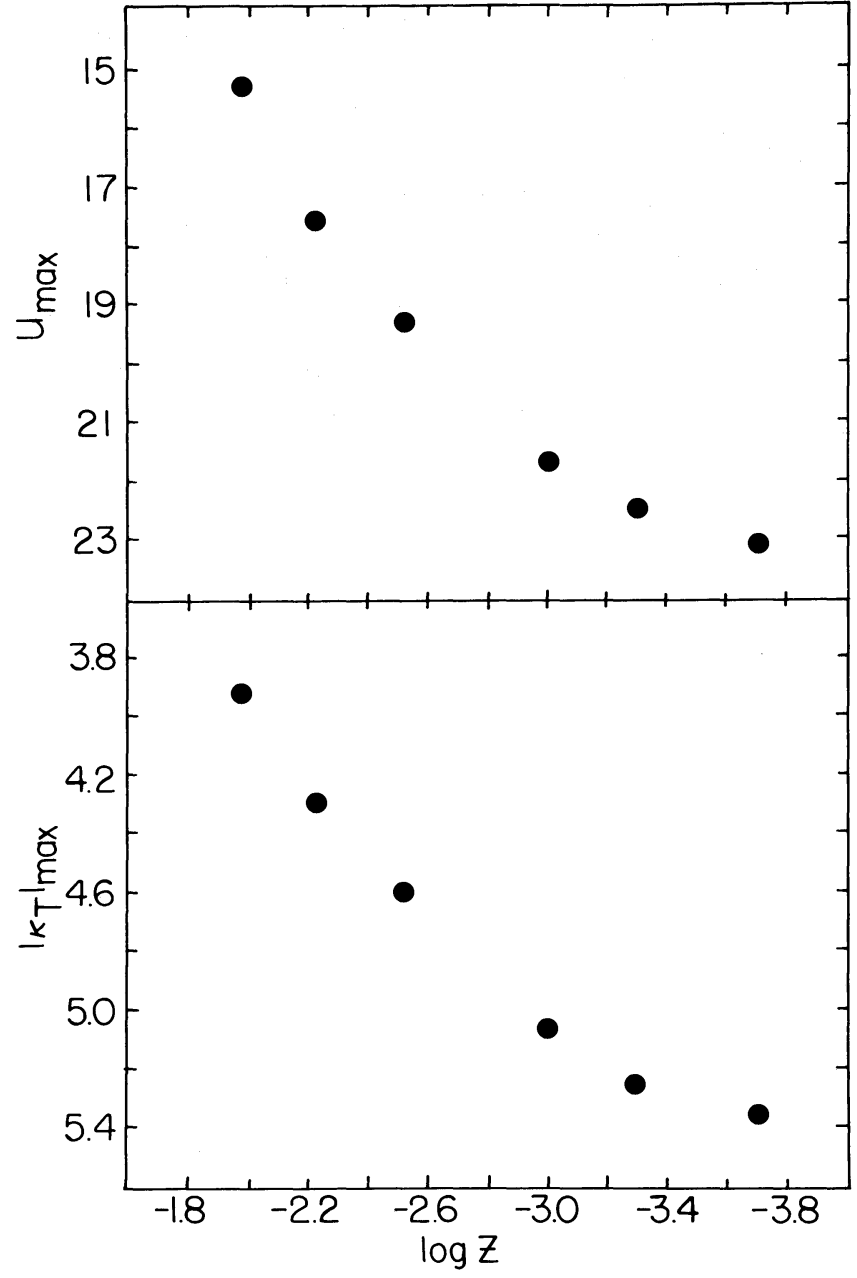

Fig. 10.--Same as Fig. 9, except that models were constructed with artificially enhanced metals opacity.

larger $u_{\max }$, the greater the average value of $u$ in the zones with $T<2 \times 10^{5} \mathrm{~K}$ which comprise the adiabatic onset domain.

Now, one notes from the one-zone calculations that $\phi_{21}$ is a monotonic decreasing function of $u$. If this result may be applied to more realistic models, then it can be seen that Figure 9 has exactly the same character as Figure $1 a$. In that case, the decrease of $\phi_{21}$ with decreasing metallicity is explained, essentially as an opacity effect. The scatter in Figure $1 a$ may be imputed to a range of mass and luminosity among the SP stars. However, the correspondence between Figures $1 a$ and 9 appears to break down somewhat in the low-metallicity regime (say $5 \leq \Delta S \leq 8,-3.3 \leq \log Z \leq-2.8$ ) where the $\phi_{21}-\Delta S$ relation retains its slope while the fall of $u_{\max }$ with $\log Z$ moderates substantially, approaching zero. The slowed decline of $u_{\max }$ is due to the fact that once $Z$ gets small enough the heavy element opacity is so negligible compared to that of hydrogen and helium that reducing $Z$ still further has little effect on the temperature exponent.

In view of new opacity calculations (Iglesias, Rogers, and Wilson 1987) which show that the Rosseland mean opacity for metals may be larger than previously thought, it is interesting to examine the effect on our models of enhancing the opacity due to heavy elements. Figure 10 portrays LNA models with parameters identical to those in Figure 9 except that the metal opacity has been augmented artificially in exactly the manner described by Simon (1982). Taking into account the compressed ordinate in Figure 10 (as compared with Fig. 9), one sees that enhancing the heavy element opacity increases the slope of the $u_{\max }-\log Z$ relation everywhere, but by progressively larger amounts as $Z$ decreases. Assuming that our explanation for Figure $1 a$ is correct, then this change is in line with observations.

While it is gratifying to be able to explain the relation between $\phi_{21}$ and $\Delta S$ among the SP stars as an opacity effect, the question of why this effect should suddenly disappear in the LP stars is much more difficult to answer. In particular, we have not been able to construct any low- $Z$, LNA models which show small values of $u_{\max }$, corresponding to the large values of $\phi_{21}$ observed in many of the LP stars. We can only conjecture that the physical structure of the longer period $R R_{a b}$ stars is such that $\phi_{21}$ is no longer principally determined in the region of adiabatic onset. For example, such might be the case when resonance effects become important, as in the classical Cepheids. Unfortunately, canonical models for the RR Lyrae stars in the LP range tend to fall between the applicable resonances $P_{2} / P_{0}=0.5$ and $P_{3} / P_{0}=0.5$. Hydrodynamic $R_{a b}$ models are also of little help in this regard since they are known to give values of $\phi_{21}$ which are much too large compared with observations (Simon and Aikawa 1986). Thus, it seems that an answer to the very interesting question of the origin of the dichotomy between the SP and LP samples must await improvements in nonlinear pulsation modeling.

Finally, we remark about differences in the appropriate values of $u$ for RR Lyrae models as given by $\operatorname{SD}(5 \leq u \leq 10)$ and in the present work $(15 \leq u \leq 23)$. Since the SD calculations were one-zone and nonlinear while the present models are multizone and linear, it is not clear what the quantitative correspondence between the two definitions of $u$ ought to be. Thus it is not surprising that some discrepancy should exist. However, it must be emphasized that our explanation for the $\phi_{21}-\Delta S$ relation does depend upon the applicability to realistic models of the one-zone result that $\phi_{21}$ decreases with $u$. To the extent that this result might not apply in the multizone case, the present conclusions in this regard will be called into question.

\section{CONCLUSION}

In the present investigation we establish a very regular decrease in the Fourier parameter $\phi_{21}$ with decreasing metal content among field $\mathrm{RR}_{a b}$ stars with period $P<0$. 575 . We also show that this relation can be tentatively explained as an opacity effect, using simple one-zone models.

However, a word of caution is in order. It seems surprising that such a tight $\phi_{21}-\Delta S$ relation should exist in view of the fact that some correlation between $\phi_{21}$ and the amplitude is known (Simon and Aikawa 1986) and that $\phi_{21}$ depends on the period for $\mathbf{R R}_{a b}$ stars in $\omega$ Cen and (very tightly) for classical Cepheids with similar pulsation properties. Other physical parameters, e.g., effective temperature or mass, might also influence $\phi_{21}$. More detailed studies are necessary to verify the present results and to further investigate the important relations between Fourier parameters and the physical properties of variable stars.

The author is pleased to acknowledge support from the National Science Foundation under grants AST-8316875 and AST-8312649. 


\section{SIMON}

\section{REFERENCES}

Simon, N. R. 1982, Ap. J. (Letters), 260, L87.

1985, Ap. J., 299, 723

Aikawa, T., Antonello, E., and Simon, N. R. 1987, Astr. Ap., 181, 25

, Butler, D., Dickens, R. J., and Epps, E. 1978, Ap. J., 225, 148.

Iglesias, C. A., Rogers, F. J., and Wilson, B. G. 1987, Ap. J. (Letters), 322, L45.

IOI Lub, J. 1977a, Astr. Ap. Suppl., 29, 345.

III - 1977b, Ph.D. thesis, University of Leiden.

Martin, W. C. 1938, Leiden Ann., Vol. 17, Part 2.

Pel, J. W. 1976, Astr. Ap. Suppl., 24, 413.

Petersen, J. O. 1984, Astr. Ap., 139, 496.

Petersen, J. O., and Hansen, L. 1984, Astr. Ap., 134, 319.

1986, Ap. J., 311, 305.

Simon, N. R, and Aikawa, T. 1986, Ap. J. 304, 249.

Simon, N. R., and Moffett, T. J. 1985, Pub. A.S.P., 97, 1078

Simon, N. R., and Teays, T. J. 1982, Ap. J., 261, 586.

Stellingwerf, R. F., and Donohoe, M. 1987, Ap. J., 314, 252 (SD)

Taam, R. E., Kraft, R. P., and Suntzeff, N. 1976, Ap. J., 207, 201.

van Herk, G. 1965, Bull. Astr. Inst. Netherlands, 18, 71.

Preston, G. W. 1959, Ap. J., 130, 507. 1971, Highlights Astr., 2, 781.

Norman R. Simon: Department of Physics and Astronomy, University of Nebraska, Lincoln, NE 68588-0111 